\title{
DUAL - LOOP FORCE - DISPLACEMENT MIXED CONTROL STRATEGY AND ITS APPLICATION ON THE QUASI - STATIC TEST
}

\author{
Cong Zeng ${ }^{1,2}$ - Bin Wu ${ }^{1,3^{*}}$ - Guoshan Xu ${ }^{1}$ - Zhen Wang ${ }^{1}$ - Tianlin Pan ${ }^{2}$
}

\author{
${ }^{1}$ Key Lab of Structures and Dynamic Behavior and Control, Ministry of Education, Harbin Institute of Technology, Harbin \\ 150090, China \\ ${ }^{2}$ School of Civil Engineering and Architecture, Northeast Electric Power University, Jilin 132012, China \\ ${ }^{3}$ School of Civil Engineering and Architecture, Wuhan University of Technology, Wuhan 430070, China
}

\begin{tabular}{l}
\hline ARTICLE INFO \\
\hline Article history: \\
Received: 18.04 .2016$. \\
Accepted: 22.02 .2017$. \\
\hline Keywords: \\
Dual-loop force-displacement control method \\
Mixed control strategy \\
Control of servo-hydraulic actuator \\
Quasi-static Test \\
Coupling of actuator and specimen \\
\hline
\end{tabular}

DOI: https://doi.org/10.30765/er.38.3.6

\section{Introduction}

The axial load has a significant effect on the quasistatic testing results $[1,2]$. An accurate simulation of the axial load during the quasi-static testing for vertical structure components, such as piers, concrete columns, and coupled shear walls, is a challenge. A simplistic approach would be to lay additional weight, but its loading capacity is restrained by the space of specimen. The control accuracy and boundary condition simulation of lifting jack with open-loop control, which is most widely used in axial loading during the quasi-static testing, is barely satisfactory. Servo-hydraulic actuators are gaining recognition as the best loading device, due to its high operational flexibility and tracking performance. When the servo-hydraulic actuators are used during structural tests, both the force control mode and displacement control mode are available. The former is preferable

\begin{abstract}
:
The Quasi-static test is a well-known powerful methodology to evaluate the seismic performance of structural components and systems. One of the most important challenges in the Quasi-static testing is to achieve precise boundary conditions, especially for the axial loading of vertical components. The requirement of synchronized displacement loading and target axial force formed a pair of contradiction. A dual-loop force-displacement mixed control strategy is proposed. The presented approach is successfully verified through the quasistatic testing for a full-scale concrete filled steel tube column. The control targets are achieved with an excellent control performance.
\end{abstract}

if the stiffness of specimen is high [3,4]. However, loading in force-control mode is risky, occasionally. When the stiffness of the loaded structure suddenly drops due to a material yield or even seriously structure damage, the stroke of actuators increases rapidly to achieve the force command. This performance may cause specimen collapse, and jeopardize the safety of testing devices and staff.

The coupling of dynamics between the actuator and specimen was observed and explained [5]. It was also indicated that the above-mentioned coupling effects become significant when multiple actuators are connected to the same specimen [6]. This phenomenon will cause difficulty with accurately simulating the boundary condition in the quasi-static testing, with many unexpected testing risks possibly appearing. To achieve the expected force command in displacement loading mode of the actuators is worth to be studied.

\footnotetext{
* Corresponding author.

E-mail address: zc_1113@126.com .
} 
Two types of mixed control methods were proposed [7]; the 'displacement-force combined control' and 'displacement-force switching control,' respectively. Control mode of the actuators in the quasi-static testing was dependent on the stiffness of the isolation bearing specimen. When the test specimen was flexible, the actuator was in displacement control, but switched to force-control once the specimen became stiff. In this method, the smooth transition in the switching process was a challenge.

An additional mixed control algorithm was also presented, which was able to achieve the stable control in the quasi-static tests of MDOF structures following the prescribed force profiles [8], although it was more likely to be used as a control strategy for its single-loop feature.

A real dual-loop mixed control strategy for coupled multi-axial loading systems, such as LBCB in UIUC was presented [9]. The signal transition of displacement and force was implemented in outerloop controller, taken from an incremental iterative approach employing the Broyden update of the stiffness Jacobian of the test specimen. The Jacobian stiffness matrix can also be updated [10].

This paper presents a dual-loop feedback control algorithm and a synchro-control strategy on axial loading actuators in the quasi-static testing. The command conversions of displacement-to-force are based on the initial stiffness. Following a demonstration of the test implement procedure, an experimental verification is performed for a full-scale concrete filled steel tube column using the Testing System with Four-bar Linkage in Harbin Institute of Technology. The experimental results confirm that the proposed method has successfully achieved high precision boundary conditions, including force command of the axial load, as well as the shear form deformation.

\section{Dual-loop force-displacement mixed control Method}

Dual-loop control in this study is defined as a combined control with outer-loop force and innerloop displacement. As mentioned above, a force command and displacement command cannot be simultaneously decomposed into single actuators. Therefore, the described herein incorporates load-todisplacement command conversion using a reciprocal initial stiffness outside control loop.

\subsection{Control architecture}

The control architecture, shown in Figure 1, and the rectangular blocks in the figure, represent data processes.

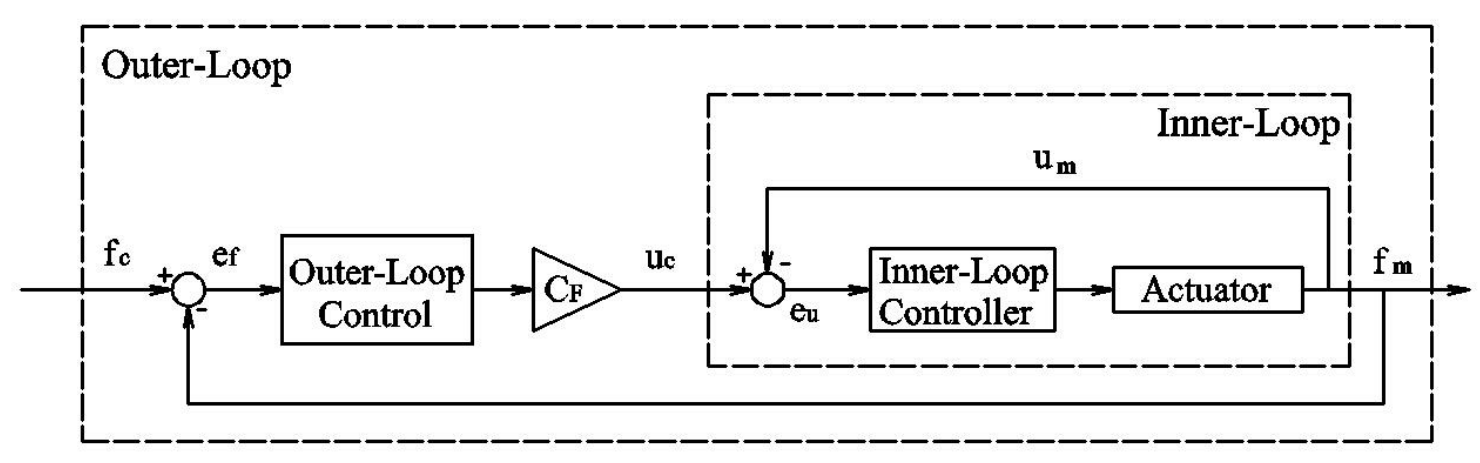

Figure 1. Block diagram for the dual-loop control.

Vectors $\mathrm{u}, \mathrm{f}$, and e are the displacement vectors, force vectors, and feedback error vectors, respectively. Superscript c, $\mathrm{m}$, f and $\mathrm{u}$ denote command, measured data, and feedback error of force and displacement, respectively. Superscript i represents the number of time steps. Finally, $\mathrm{C}_{\mathrm{F}}$ represents force-todisplacement conversion factor, which is equal to reciprocal of the initial stiffness of specimen. In the command execution process, force command is converted into a displacement command, which is decomposed into the actuator. In the measurement process, results from LVDT on actuators are used to feedback in inner-loop by the control of analog PID, and the outer-loop feedback is implemented by load cell on the actuators, which is controlled by digital PI. A brief description of each process is as follows:

1) Force-to-Displacement Conversion: the target force is converted into displacement command of actuators, using the reciprocal of the approximation of the initial stiffness, which is measured before the 
test. However, the control effectiveness is not sensitive to the initial stiffness because of the digital PI controller. It is observed that the outer-loop is robust to the measure.

2) Feedback: Measured actuator displacement and force from the LVDT and load cell are used to feedback in inner-loop and outer-loop separately.

3) Digital PI Loop: A discrete proportional-integral (PI) controller is designed to implement the outerloop feedback control and improve the control performance.

4) Analog PID Loop: An analog proportionalintegral-derivative (PID) (inner) control loop is employed for a continuous feedback for each actuator.

\subsection{Mixed control strategy for axial loading in the quasi-static Testing}

For the global stability of loading system in the quasistatic testing, the number of axial loading actuators is always greater than two. The axial boundary condition cannot be achieved with independent control of each actuator. Therefore, a mixed control strategy for multiple actuators is proposed. The block diagram is shown in Figure 2.

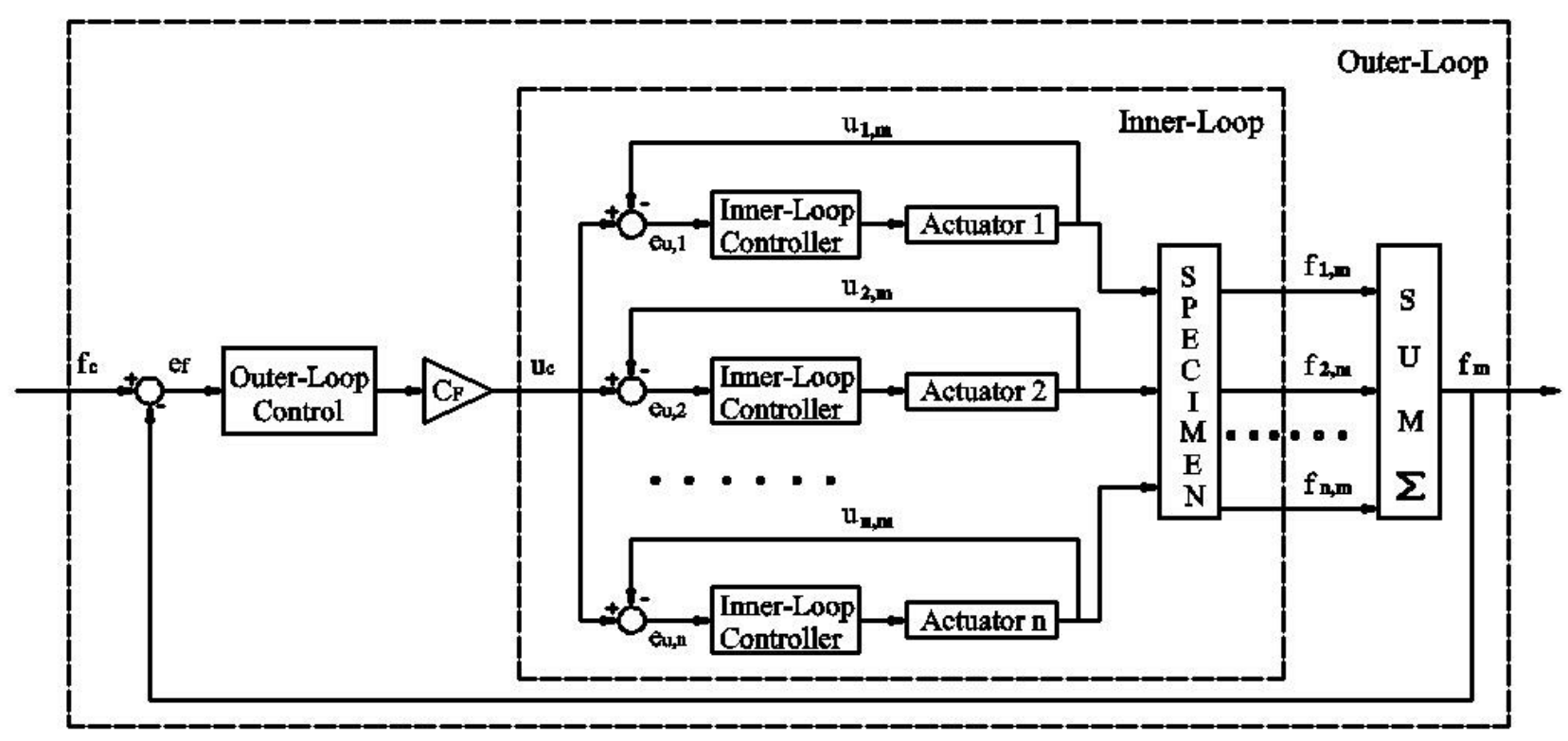

Figure 2. Block diagram for the coupled control strategy

The definition for the letters in Fig.2 is similar to the former section, and the numbers in superscript denote the identifier of the actuators. The target command is the resultant axial force fc, and the outer-loop feedback is the summary of all the actuators' load cells. The feedback error of outer-loop $e_{f}$ is transferred to the execution command of each actuator, which is in the displacement control mode. It is obvious that not only the value of all the execution command for each actuator is the same, but also they are synchronous under the identical innerloop control.

\subsection{Control performance of single actuator}

To test the control performance, validation tests are carried out (Shown as Figure 3). In the test of dual- loop control, the controller design is the key part, especially for the outer-loop digital PI controller. The value of $\mathrm{P}$ and I can be determined by numerical modeling and simulation. The transfer function with the consideration of actuator-specimen interaction can be written as follows [11]:

$$
H(s)=\frac{2 \beta_{e} k_{0} A_{p}}{M_{e} V_{t} s^{3}+C_{e} V_{t} s^{2}+\left[K_{e} V_{t}+4 \beta_{e} A_{p}^{2}\right] s}
$$

The definition of parameters in the above equation and the value in numerical simulation are listed in Tab.1.

Firstly, the numerical model of the test system is established and simulated in Matlab. The P and I gains are selected by the modification and 
optimization on the parameters of PI controller as follows:

1) outer-loop controller: $P$ gain $=0.05$, I gain $=2$;

2) inner-loop controller, $\mathrm{P}$ gain $=5$, I gain $=1$, which were pre-installed in the factory.

Table 1. System Parameters of simulation

\begin{tabular}{|c|c|c|c|}
\hline & Parameters & value & unit \\
\hline $\mathrm{k}_{0}$ & $\begin{array}{l}\text { Gain coefficient of servo } \\
\text { valve }\end{array}$ & 1.0674 & $\mathrm{~m}^{2} / \mathrm{s}$ \\
\hline$A_{p}$ & Area of piston & 487.7 & $\mathrm{~cm}^{2}$ \\
\hline $\mathrm{V}_{\mathrm{t}}$ & Volume of actuator & 15522 & $\mathrm{~cm}^{3}$ \\
\hline$\beta_{\mathrm{e}}$ & $\begin{array}{c}\text { Compression modulus of } \\
\text { hydraulic liquid }\end{array}$ & 700 & Mpa \\
\hline $\mathrm{K}_{\mathrm{e}}$ & $\begin{array}{l}\text { Initial stiffness of } \\
\text { specimen }\end{array}$ & 14500 & $\mathrm{kN} / \mathrm{m}$ \\
\hline $\mathrm{M}_{\mathrm{e}}$ & $\begin{array}{l}\text { Equivalent mass of } \\
\text { specimen }\end{array}$ & 24500 & $\mathrm{~kg}$ \\
\hline$\zeta$ & $\begin{array}{l}\text { Damping ratio of } \\
\text { specimen }\end{array}$ & 0.02 & 1 \\
\hline $\mathrm{CF}$ & $\begin{array}{l}\text { Conversion coefficient of } \\
\text { force-to-displacement }\end{array}$ & $1 / 14500$ & $\mathrm{~m} / \mathrm{kN}$ \\
\hline
\end{tabular}

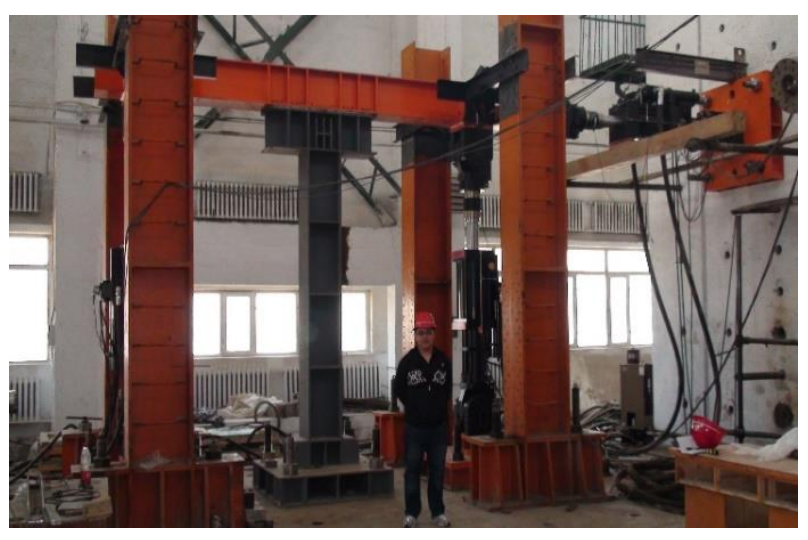

Figure 3. Test setup of 3 DOF testing system in HIT.

To test the control performance in a single actuator, the lateral actuator is used. The step response and sine response are shown in Figure 4. As can be seen in the test, the actuator correctly executes the outer-loop force command. Although the response rate is not quick enough, it is acceptable for a 0.5 second delay in the slow quasi-static tests.

\subsection{Control Performance in Multiple Actuators}

The two vertical actuators are controlled by the proposed mixed control strategy, as seen in Fig. 3. The specimen is a full-scale steel column and the target axial force is $100 \mathrm{kN}$. The lateral loading in the quasi-static testing can be considered as a periodic perturbation. To test the robustness, the lateral actuator executed a sine wave with the amplitude of $50 \mathrm{kN}$ and frequency of $0.1 \mathrm{~Hz}$. The controllers of the vertical actuators are the same as the above section. The control performance can be seen in Figure 5.

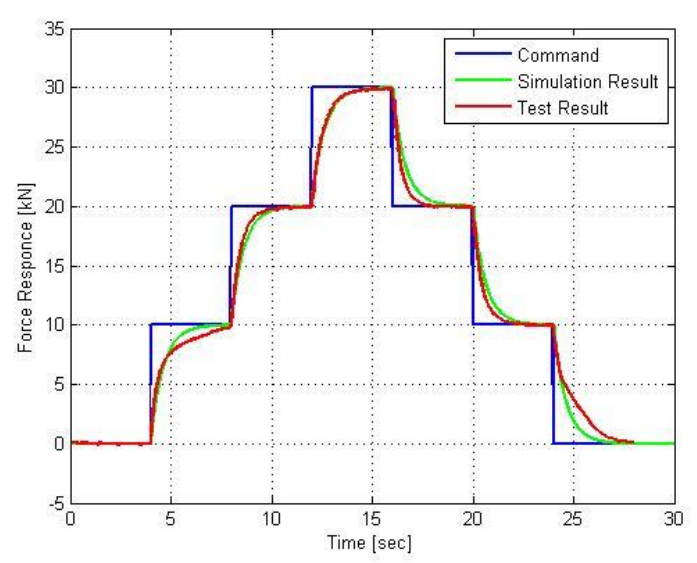

(a) step response

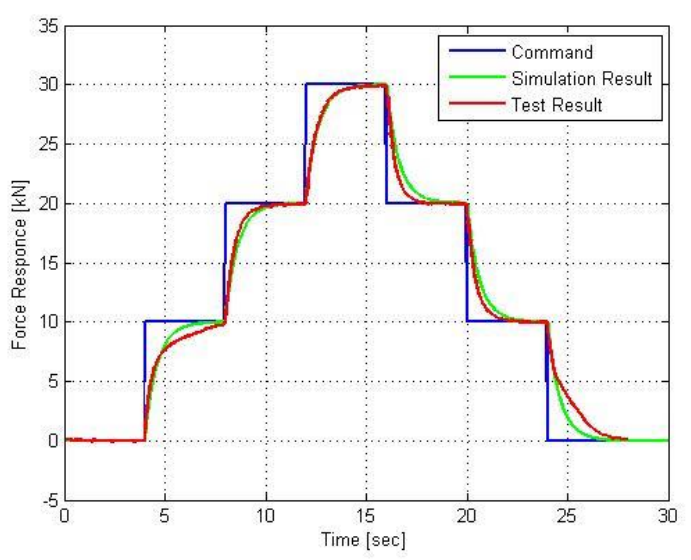

(b) sine response

Figure 4. Performance of dual-loop control method applyed in single actuator.

The test results show that coupled with deformation of the lateral actuator, the force response of each vertical actuator stays within the range of $20 \%$, but the resultant force responses are only varied in the range of no more than $1.2 \%$ under the control of the proposed strategy. Moreover, the most important thing is that the displacement of two vertical actuators always stays in synchrony throughout the test, which leads to the achievement of shear-form loading. Additionally, the coupling effect between the vertical and lateral actuators truly exists, but the 
influence is negligible in the slow-rate quasi-static tests.

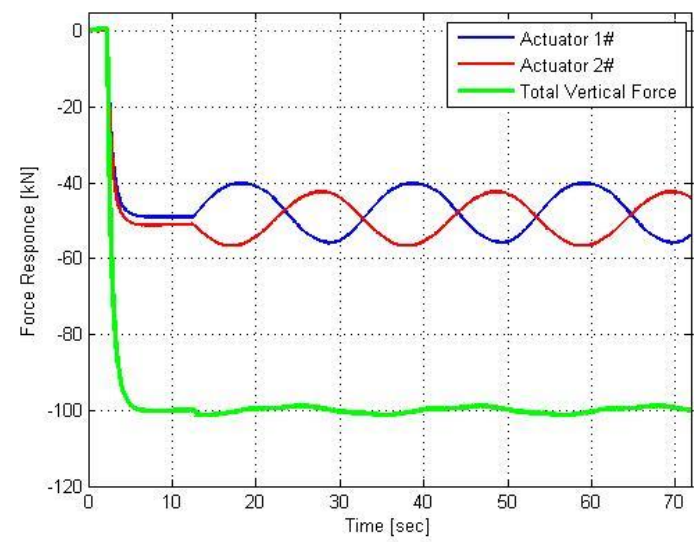

(a) force response

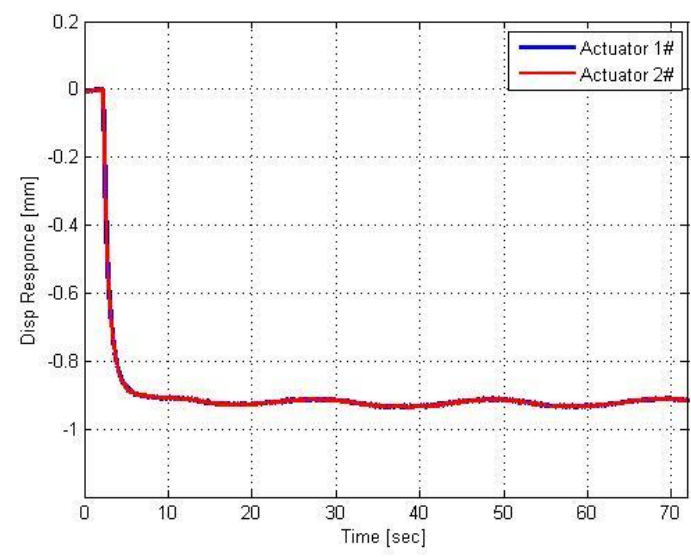

(b) displacement response

Figure.5 Results of the Validate Quasi-static Test.

\section{Experimental verification}

\subsection{Test Setup and loading scheme}

As shown in Figure 6 and Figure 7, the test is conducted by the Testing System with Four-bar Linkage in HIT, which includes a reaction wall, a loading frame, a L-shape loading beam, two vertical actuators, a horizontal actuator, PC for control and operate, oil sources, and controller. The specimen, a full-scale regenerated concrete filled steel tube column, is tied to the test bed. The two vertical actuators, which are installed between the loading frames and loading beams are used to apply constant vertical forces to the columns under the control of proposed mixed control strategy. The horizontal actuators, which are fixed to the reaction wall in the height of inflection point of specimen, are used to apply the horizontal forces under traditional singleloop force control.

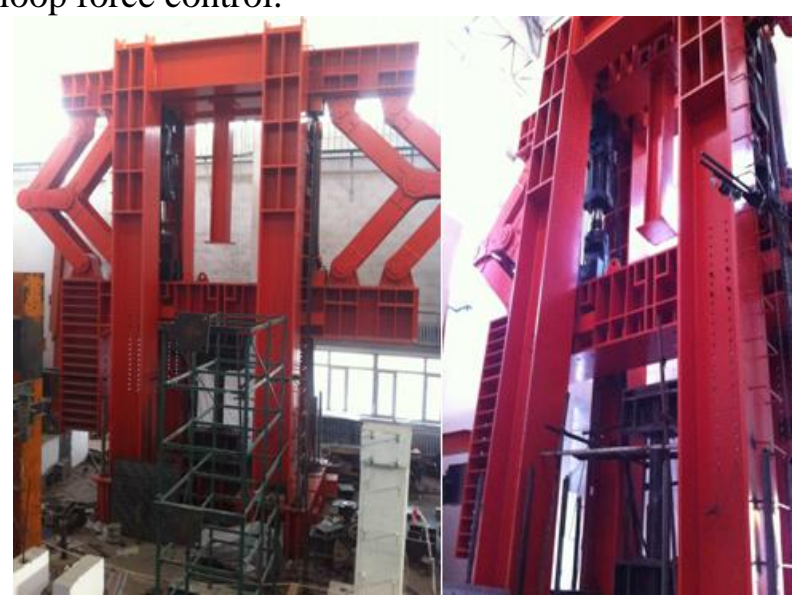

Figure 6. Photo of the Testing System with Four-bar Linkage in HIT.

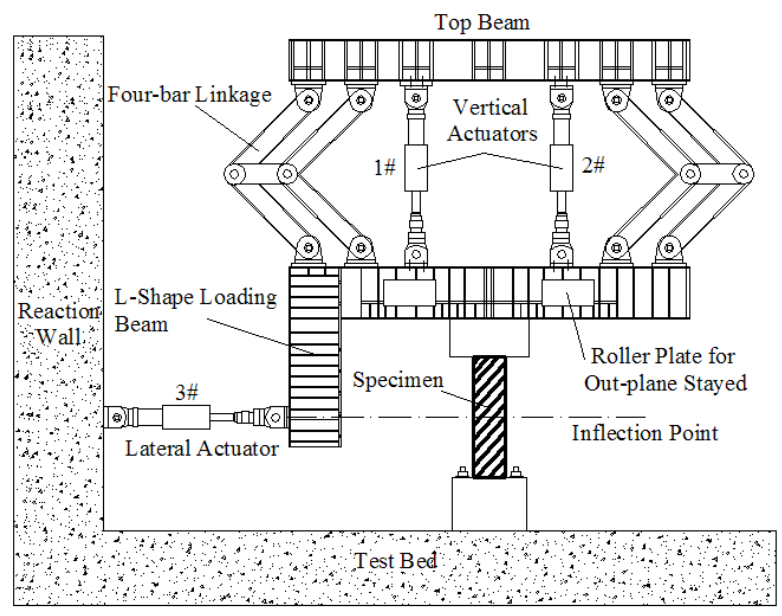

Figure 7. Outlines of the Testing System with Fourbar Linkage in HIT

The loading scheme for the lateral actuator is shown in Figure 8. The load follows a triangular pattern with an incremental amplitude. All the actuators, which are quasi-static with loading capacity of $2000 \mathrm{kN}$ in compression and $1400 \mathrm{kN}$ in tension, are part of the servo-hydraulic loading system made by MTS Systems Corporation. The stroke of all the actuators is $\pm 250 \mathrm{~mm}$. The control accuracy for both force and displacement is $0.5 \%$ of the full span. The test is controlled based on the FlexTest 60 and test program MTS793.10, which are the hardware and software test platforms provided by MTS Corporation and ran on the Microsoft Windows system.

The detailed introduction on the procedure of the quasi-static test, which is controlled by the proposed strategy, is as follows: 
1) Step 1: After the channels of the vertical actuators are established in Station Builder, calculation channels are needed. The specific operation method is to add ' $<<$ Calculated $>>$ ' from 'input hardware resource' in the 'External Command' toolbar. Actuator 1\# and 2\# represent the two vertical actuators separately.

2) Step 2: Pick the 'Calculation Editor' from the 'Tools' menu under the 'Configuration' authority in Station Manager. Then, write and debug the external command program in calculation editor. The specific program used in this test can be downloaded on our web set, http://hytest.hit.edu.cn.

3) Step 3: Use the MPT (MultiPurpose Testware) in the Application menu of Station Manager under the 'Operator' authority. Add the programmed external command to the process as the control command of the vertical actuators. The 'Run-time Ramp Control' is also added to execute the lateral actuator. In order to obtain the test results, the process of data collection and figure plotting also had to be put into the process sequence.

4) Step 4: Manually operate the vertical actuators under displacement control mode to ensure the horizontal loading beam by aid of the leveling tube or instrument.. Then, the axial load can be exerted to the specimen by using MPT case introduced in the step 3. After the axial load is stable, the tools of Runtime Ramp Control can also implement vertical loading.

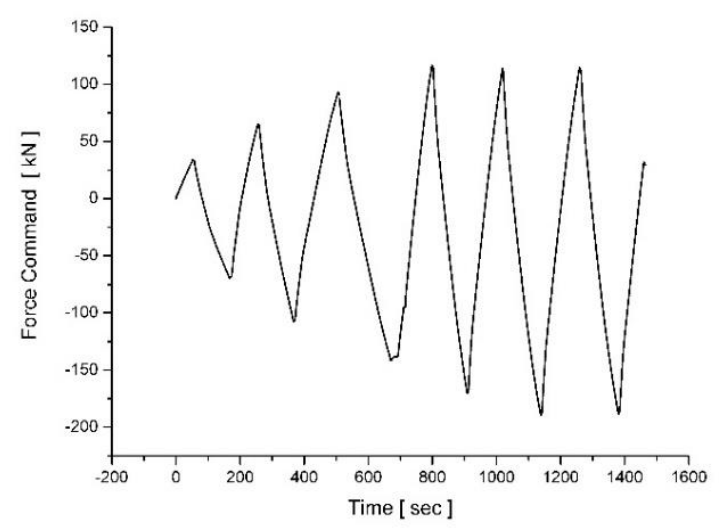

Figure 8. Loading Scheme of the Lateral Actuator.

\subsection{Test Results}

For vertical loading in the quasi-static test, two control targets are required:

1) Holding stable axial resultant force during the whole test.
2) Shear-form loading, in other words, the loading beam should keeping horizontal during the test.

The main purpose of the test is to validate whether the control target can be achieved. The force and displacement responses are shown in Figure 9 and Figure 10.

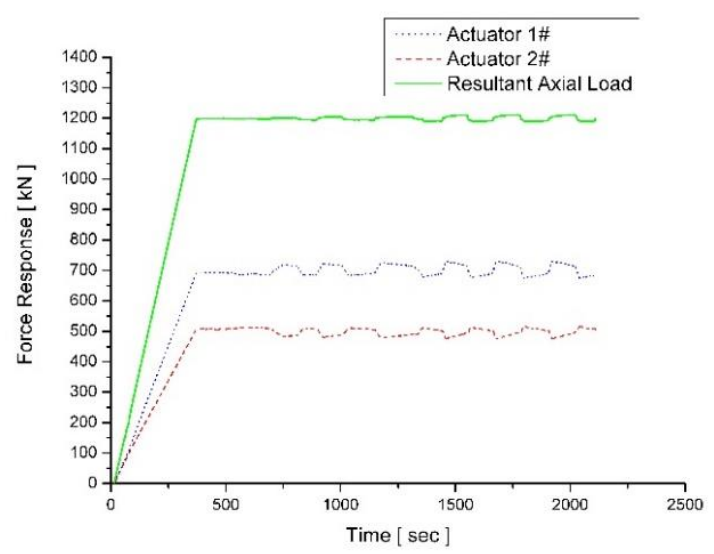

Figure 9. Force response of the vertical actuators.

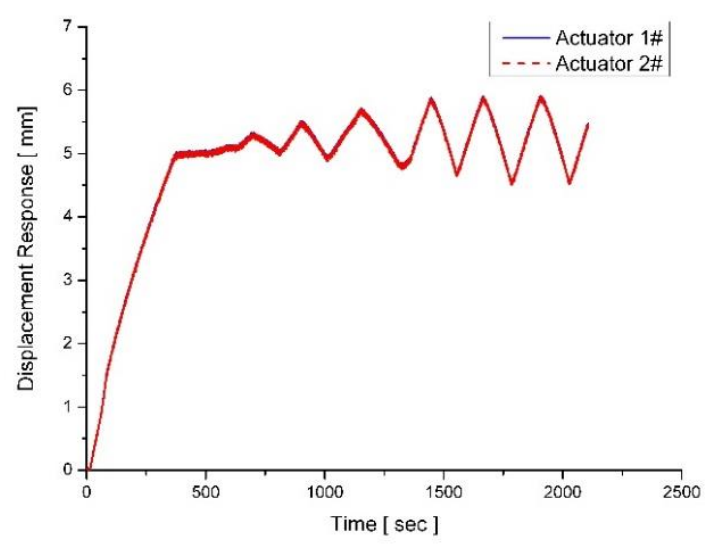

Figure 10. Displacement response of the vertical actuators

The test results reveal that the control target of the vertical actuators is successful. Under the lateral loading speed of $0.01 \mathrm{~mm} / \mathrm{s}$, , the mean percent error of axial resultant force is only $0.8 \%$ in 25 mins' test, and the root-mean-square percent error is also less than 5\%. The loading precision can be fully acceptable in the quasi-static tests. Importantly, the shear-form loading is perfectly implemented, as shown in Figure 10. The difference of displacement response between the two vertical actuators is no more than $0.02 \mathrm{~mm}$ during the test, which means the loading beam is keeping horizontal throughout the test. The control performance of the proposed mixed control strategy is excellent. Only 
the precise boundary condition can ensure that the test results are reliable and credible. The specific test results will be introduced in other studies.

\section{Conclusion}

In this study, a force-displacement dual-loop mixed control method is proposed in order to achieve a precise simulation for the axial loading boundary conditions of the structural vertical components in the quasi-static tests. The algorithm was applied to the quasi-static test of a full-scale concrete filled steel tube column to investigate its effectiveness. The major conclusions obtained from this study are as follows:

1) The dual-loop mixed control, which combined outer-loop force control and inner-loop displacement control, is a simple and effective control approach. By applying the algorithm to the quasi-static tests, the stable axial force and the shear-form loading can be simultaneously calculated.

2) The design of outer-loop controller is partly dependent on the model of the actuator-specimen system, although the P and I gain can be easily obtained by several times of attempts and adjustments. The system is robust, and is not sensitive to the force-displacement transition factor. 3) The dual-loop control method is applicable in more ways than just the control of vertical actuators in the quasi-static tests. Moment exertion on the wall and column components, lateral proportional loading, and loading on the specimen with stiffness degradation may all be the future applications of the method. Further studies will be developed.

\section{Acknowledgements}

The financial support from the National Key Research and Development Program of China under Grant No. 2016YFC0701106, Natural Science Foundation of China under Grant Nos. 51161120360, 51778190, and the Department of Science and Technology of Jilin Province of China under Grant No. 20150623024TC-16 is gratefully acknowledged.

\section{References}

[1] Abrams, D. P.: Influence of axial force variation on flexural behavior of reinforced concrete columns. ACI Struct. J., 5-6 (1987), 246-254.

[2] Chen, Y., Wu, B., Pan, T.: A Novel Sectional Constitutive Model for Beam-column Element. Engineering Review, 36 (2015),41-52.

[3] Elwood, K. J., Moehle, J. P.: Dynamic collapse analysis for a reinforced concrete frame sustaining shear and axial failures. Earthquake Engineering and Structural Dynamics, 37 (2008), 991-1012.

[4] Shoraka, M. B.: Hybrid Simulation of the Gravity Load Collapse of Reinforced Concrete Frames, $18^{\text {th }}$ Analysis and Computation Specialty Conference (ASCE), Wisconsin USA, 2008.

[5] Dyke, S. J., Spencer, B. F., Jr., Quast, P., and Sain, M. K.: Role of control-structure interaction in protective system design. J. Eng. Mech.,1995,121 (2), 322-338.

[6] Phillips, B. M.: Model-Based Multiactuator Control for Real-Time Hybrid Simulation. J. Eng. Mech.139 (2013),2, 219-228.

[7] Pan, P., Nakashima, M., Tomofuji, H.: Online test using displacement-force mixed control. Earthquake Engineering and Structural Dynamics,34 (2005), 869-888.

[8] Pan, P., Zhao, G., Lu, X., Deng, K.: Forcedisplacement mixed control for collapse tests of multistory buildings using quasi-static loading systems. Earthquake Engineering and Structural Dynamics, 43 (2014), 2, 287-300.

[9] Nakata, N., Spencer, B. F., Elnashai, A. S.: Multi-dimensional hybrid simulation using a six-actuator self-reaction loading system, The $14^{\text {th }}$ World Conference on Earthquake Engineering, Beijing, China, 2008.

[10] Mahmoud, H. N., Elnashai, A. S., Spencer, B. F., Kwon, O., Bennier, D. J.: Hybrid simulation for earthquake response of semirigid partialstrength steel frames. Journal of Structural Engineering, 139 (2013), 7, 1134-1148.

[11] Jung, R.: Development of real-time hybrid test system. PhD. Thesis. University of Colorado, 2005. 